\title{
Semiautomatic, Quantitative Measurement of Aortic Valve Area Using CTA: Validation and Comparison with Transthoracic Echocardiography
}

\author{
V. Tuncay, ${ }^{1}$ N. Prakken, ${ }^{2}$ P. M. A. van Ooijen, ${ }^{1,2}$ \\ R. P. J. Budde, ${ }^{3}$ T. Leiner, ${ }^{3}$ and M. Oudkerk ${ }^{1}$ \\ ${ }^{1}$ Center for Medical Imaging North East Netherlands (CMI-NEN), Department of Radiology, University of Groningen, \\ University Medical Center Groningen, P.O. Box 30001, 9700 RB Groningen, Netherlands \\ ${ }^{2}$ Department of Radiology, University of Groningen, University Medical Center, P.O. Box 30001, 9700 RB Groningen, Netherlands \\ ${ }^{3}$ Department of Radiology, University Medical Center Utrecht, P.O. Box 85500, 3508 GA Utrecht, Netherlands
}

Correspondence should be addressed to P. M. A. van Ooijen; p.m.a.van.ooijen@umcg.nl

Received 12 September 2014; Accepted 3 April 2015

Academic Editor: Michael Gotzmann

Copyright (C) 2015 V. Tuncay et al. This is an open access article distributed under the Creative Commons Attribution License, which permits unrestricted use, distribution, and reproduction in any medium, provided the original work is properly cited.

\begin{abstract}
Objective. The aim of this work was to develop a fast and robust (semi)automatic segmentation technique of the aortic valve area (AVA) MDCT datasets. Methods. The algorithm starts with detection and cropping of Sinus of Valsalva on MPR image. The cropped image is then binarized and seed points are manually selected to create an initial contour. The contour moves automatically towards the edge of aortic AVA to obtain a segmentation of the AVA. AVA was segmented semiautomatically and manually by two observers in multiphase cardiac CT scans of 25 patients. Validation of the algorithm was obtained by comparing to Transthoracic Echocardiography (TTE). Intra- and interobserver variability were calculated by relative differences. Differences between TTE and MDCT manual and semiautomatic measurements were assessed by Bland-Altman analysis. Time required for manual and semiautomatic segmentations was recorded. Results. Mean differences from TTE were -0.19 (95\% CI: -0.74 to 0.34$) \mathrm{cm}^{2}$ for manual and -0.10 (95\% CI: -0.45 to 0.25$) \mathrm{cm}^{2}$ for semiautomatic measurements. Intra- and interobserver variability were $8.4 \pm 7.1 \%$ and 27.6 $\pm 16.0 \%$ for manual, and $5.8 \pm 4.5 \%$ and $16.8 \pm 12.7 \%$ for semiautomatic measurements, respectively. Conclusion. Newly developed semiautomatic segmentation provides an accurate, more reproducible, and faster AVA segmentation result.
\end{abstract}

\section{Introduction}

Aortic stenosis (AS) is the most common valvular heart disease in the developed countries, affecting 3 percent of the population older than 65 years. It causes higher morbidity and mortality than any other cardiac valve disease [1]. AS is defined as narrowing of the aortic valve opening, which reduces blood flow from the heart into the aorta. The normal size of the aortic valve area (AVA) at maximum opening of the valve is 3 to $4 \mathrm{~cm}^{2}$ [2]. When the AVA decreases below $1 \mathrm{~cm}^{2}$, AS is considered to be severe [3]. For severe AS, valve replacement is the only effective treatment. However, a sizeable fraction of patients are at high risk for postoperative mortality and may refuse surgery or cannot undergo surgery due to comorbidity [4]. Recently, transcatheter aortic valve replacement (TAVR) techniques have been developed to provide less invasive treatment for those patients [5-11]. In management of AS, the timing for surgical treatment is very important. Late treatment may lead to an increase in the transaortic pressure gradient, myocardial pressure overload, and eventually left ventricular (LV) hypertrophy and increased LV wall thickness [12]. Visualization of the AVA is used to determine the threshold for invasive treatment and to obtain preoperative information about the aortic dimensions and proximity to other important structures such as the coronary arteries.

Different imaging modalities have been used and compared for measuring the AVA [13-23]. Initially, catheterization was the standard method for evaluating AVA, but in time its usage decreased due to its being an invasive modality 
and technical limitations. Alternatively, the 2D echocardiographic continuity equation, which currently is the most common tool to derive the AVA, was used to measure the AVA. However, this technique underestimates AVA since it assumes that the left ventricular outflow tract (LVOT) has circular geometry and that flow through the LVOT is laminar and uniform [24, 25]. Bruder et al. showed a strong correlation between the AVA determined by echocardiography and MRI [20] indicating that MRI can also be used to determine AVA. However, MRI is contraindicated for patients with metal implants or claustrophobia. Moreover MRI has lower spatial resolution in comparison to CT [12]. More recently, development of ECG-gated multidetector computed tomography (MDCT) has led to further improvements in cardiac imaging [21], and CT is now also regarded as a reliable method to measure the AVA [26]. The latest developments in dual source and 320 -slice CT enable high temporal resolution acquisition and obtain sufficient image quality at high spatial resolution in almost every patient throughout the cardiac cycle. However, streaking and blooming artifacts due to heavy calcification of the aortic valve leaflets or the aortic root which hamper visualization and analysis of the AVA can still occur.

Delgado and Bax suggested that 3D planimetric measurement of the AVA by MDCT images may provide more reliable information on the assessment of AVA in comparison with echocardiography [27]. However, planimetric measurement of AVA is currently performed manually by the radiologist using standard $3 \mathrm{D}$ visualization and measurement software, which is time consuming and introduces user dependence and intra- and interobserver variability [28].

The aim of this study was to develop and validate a (semi)automatic segmentation technique of the AVA and to compare manual and semiautomatic measurements with the Transthoracic Echocardiography (TTE) results. Our goal is to reduce the user dependency and time spent on measurements and to enable reproducible and accurate measurement of AVA on MDCT datasets.

\section{Materials and Methods}

2.1. Experimental Design. In this study multiphase CT scans of 25 patients ( 15 female, mean age $82.84 \pm 5.16$ years) were used. All of the patients had moderate to severe aortic stenosis and underwent TAVI at a tertiary referral center. All subjects underwent CT scanning and TTE.

Informed consent requirement was waived by the local IRB because of the retrospective nature of this study without additional burden to the patients involved.

The maximum aortic valve opening phase was selected visually for all patients. A stack of reformations was obtained after centering the axis of the multiplanar reconstruction (MPR) at the level of aortic valve and then changing the orientation of the plane perpendicular to the LVOT. The preselected slices were segmented both manually and semiautomatically by two independent observers 1 , a biomedical engineer with more than 5 years of experience, and 2, a cardiac radiologist with almost 10 years of medical imaging experience. Observer 1 repeated the measurement 1 day after in order to determine intraobserver variability. Manual and semiautomatic measurements were compared with the current reference standard Transthoracic Echocardiography (TTE) with regard to AVA. Time spent for measurements was recorded for manual and semiautomatic segmentations.

2.2. Transthoracic Echocardiography. TTE was performed as part of the routine workup of the patient. TTE derived AVA measurements were obtained from the clinical patient records. The velocity in the left ventricular outflow tract and at the level of the aortic valve and the LVOT diameter were measured. From these measurements the AVA was calculated using the continuity equation.

2.3. Multislice Computed Tomography. Image acquisition of the retrospectively ECG-gated CTA of the thoracoabdominal aorta was performed on a multidetector 256-slice CT (Brilliance iCT, Philips Healthcare, Best, The Netherlands). An ECG trace was recorded during the procedure. The region of acquisition ranged from above the aortic arch to the groin. Based on a locator image, a circular region of interest was drawn within the descending aorta. Nonionic iodinated contrast material (Ultravist, $300 \mathrm{mg}$ iopromide per mL, Schering Nederland BV, Weesp, The Netherlands) was injected intravenously. As soon as the descending aorta reached a density of 125 Hounsfield units (HU) within the region of interest, the patient was instructed to maintain a breath hold. Seven seconds later, image acquisition started in a craniocaudal direction with concurrent ECG trace recording. The following parameters were used: detector collimation $128 \times 0.625 \mathrm{~mm}$; pitch 0.30 ; matrix size $512 \times 512$. Tube voltage and tube current-time product depended on the patient's weight and were $100 \mathrm{kVp}$ and $300 \mathrm{mAs}$, respectively, for patients $<70 \mathrm{~kg}$, and $120 \mathrm{kVp}$ and $250 \mathrm{mAs}$, respectively, for patients $\geq 70 \mathrm{~kg}$.

2.4. AVA Segmentation Algorithm. The segmentation algorithm (Figure 1) starts with the detection of the Sinus of Valsalva (SOV) on the MPR image. Once the SOV is detected, the region covering the $\mathrm{SOV}$ is cropped from the whole image (Figure 1(a)). The cropped image (Figure 1(b)) is binarized by adaptive thresholding (Figure $1(\mathrm{c})$ ). The user places seed points (Figure 1(d)) to create an initial contour (Figure 1(e)) covering the aortic valve opening. The contour moves towards the edge of the aortic valve opening automatically (Figure 1(f)). The contour covers the pixels from the edge of the opening area and also the AVA opening area. The pixels of the opening area are selected and the number of pixels is multiplied by the pixel size to determine the AV opening area (Figure $1(\mathrm{~g})$ ).

2.4.1. Detection and Cropping of the Sinus of Valsalva. Image cropping was used to reduce computation time. In the object detection the object size, shape, location, and orientation play major roles. Since the SOV is located in the central part of the MPR image, detection and cropping of this region begin with a preliminary cropping operation, which covers the most of the central part of the MPR image. After the initial cropping, the grayscale image is binarized using global thresholding with a threshold level based on the histogram 

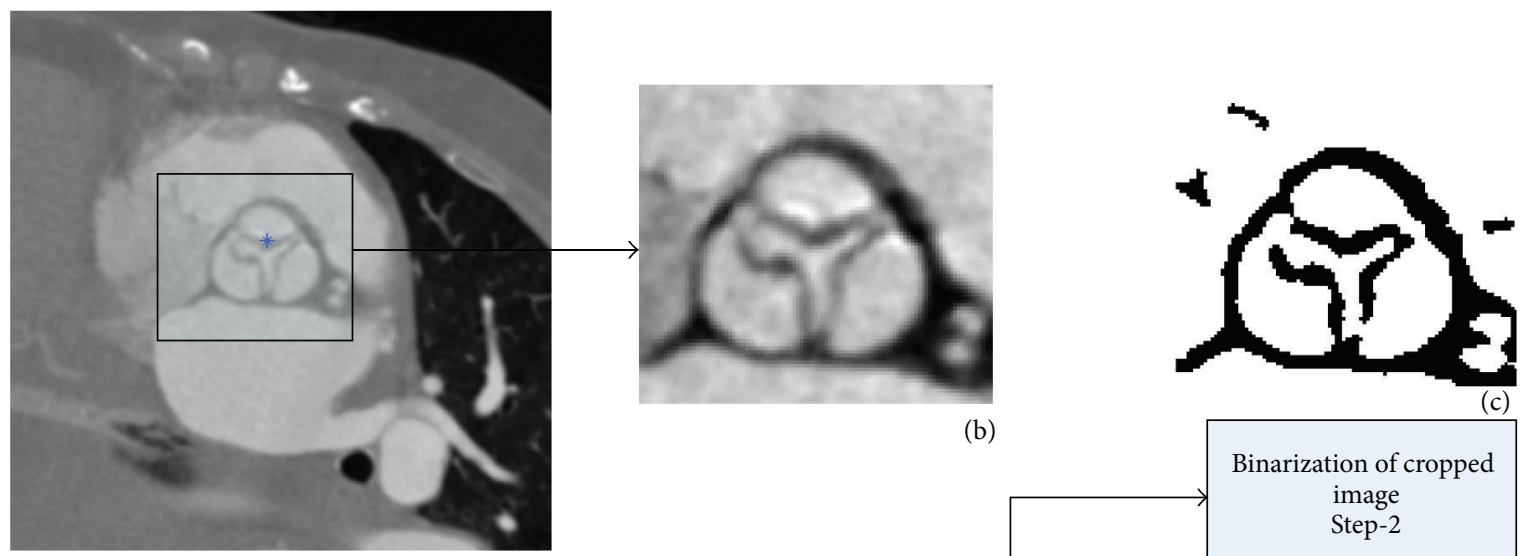

(b)

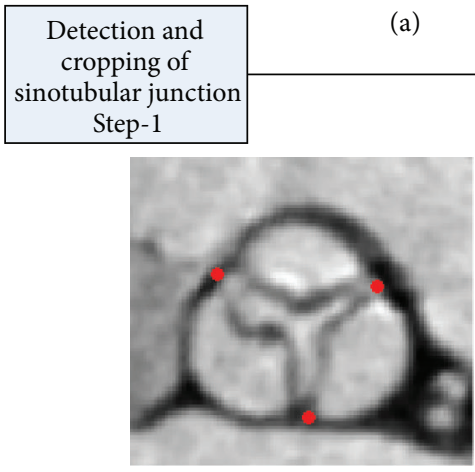

(d)

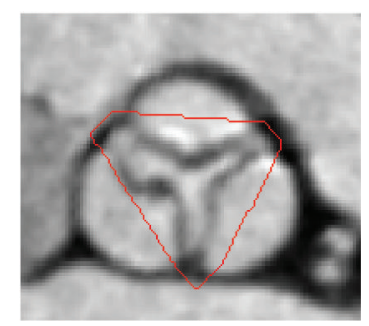

(e)
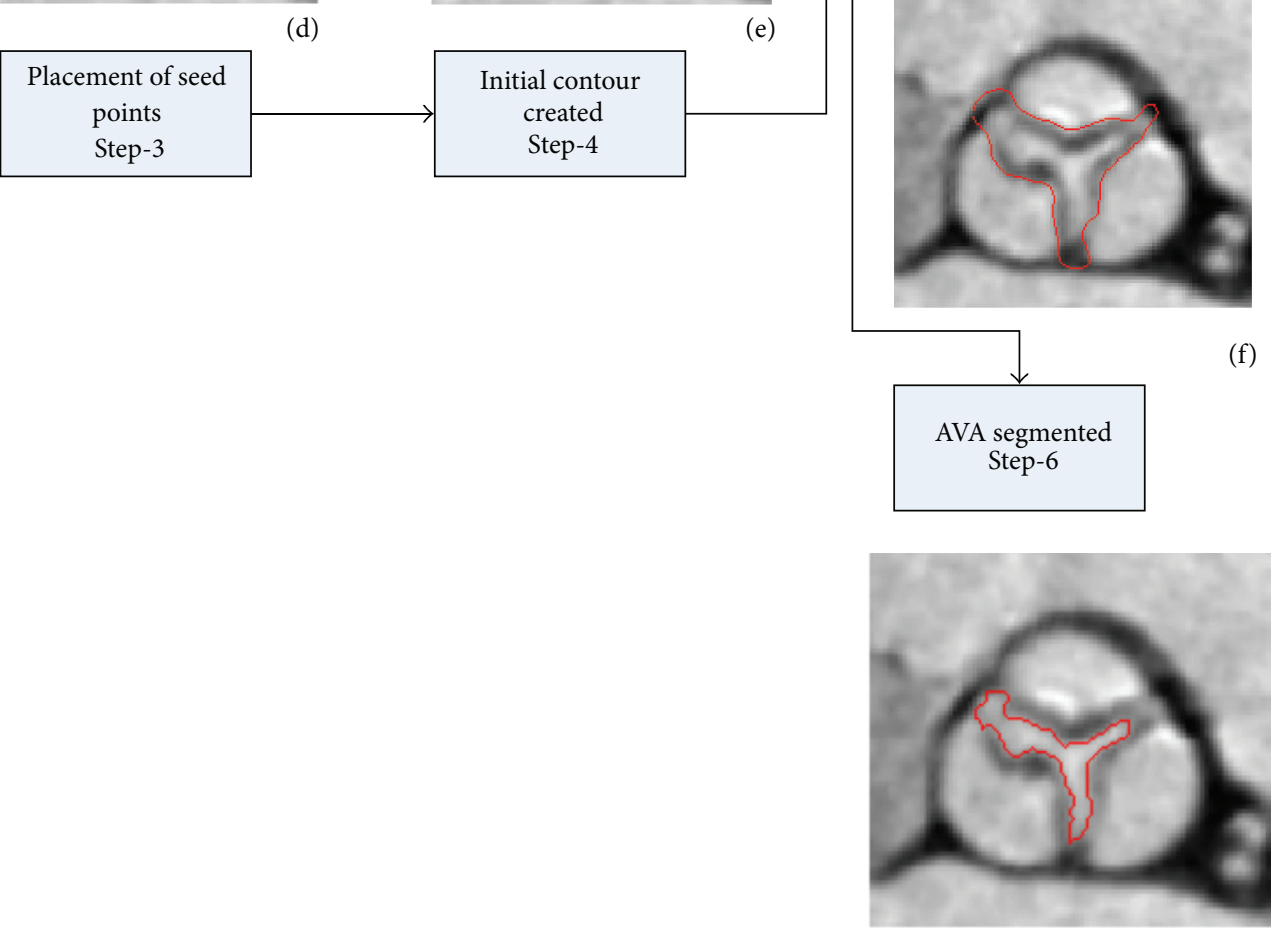

(g)

FIgURE 1: The flowchart of the general algorithm.

of the image. The binary image contains only white (object) and black (background) pixels, which enables detection of the objects in the image and facilitates the use of morphological operations. Following binarization, objects smaller than 700 pixels were removed. Secondly the SOV was disconnected and isolated. The SOV is located in the central part of image, such that objects on the border of the image were removed. After detection of the SOV, the region covering it was cropped 


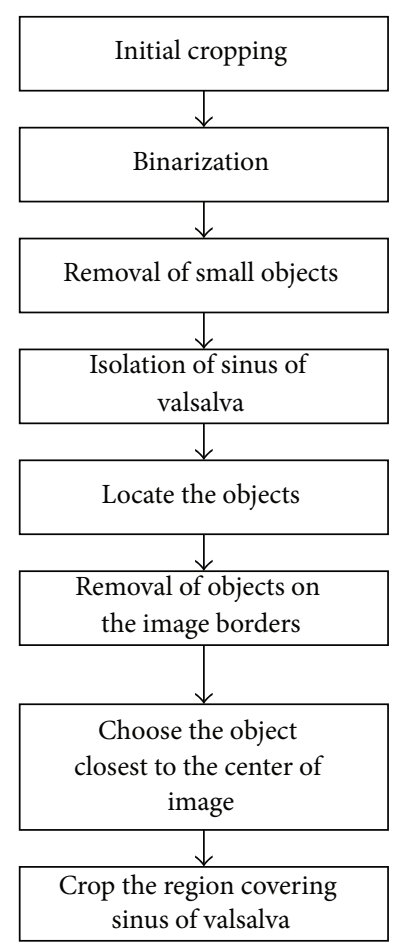

FIGURE 2: The flowchart of the SOV detection and cropping algorithm.

from the image (Figure 2). All following operations were performed on the cropped image.

2.4.2. Segmentation of AVA. The flowchart in Figure 3 shows the details of the segmentation of the AVA. The main segmentation tool is the gradient vector flow (GVF) snake [29]. The snake algorithm is an active contour, which moves to the edges of the object in order to reach the boundaries of the object. In the binarized images (described above) the edges are clearer and the active contour can move towards the object boundaries easier compared to grayscale images. The user places three seed points on the grayscale image where the cusps are connected to each other to create the initial contour for the GVF snake. This initial contour creates a mask image, which is used on the binarized image. The active contour shrinks to cover the AVA region. However, the GVF snake result overestimates the AVA. Therefore this GVF snake contour is used to mask the image again. In case the resulting double-masked image contains more than 1 object, the size and location of these objects were determined. Objects smaller than $40 \mathrm{~mm}^{2}$ and the object most distant from the center were removed. The remaining object was identified as the AVA.

2.4.3. Detection and Removal of Calcification. Aortic valve calcification is very common in a population with AS. In order to segment the AVA properly one must first detect the calcifications and then exclude the calcified areas from the AVA region. Since a contrast agent was used in the CT scans we cannot use the fixed $130 \mathrm{HU}$ threshold to detect pixels in calcified areas. We therefore developed an algorithm to determine the threshold of calcified pixels, consisting of the following 5 steps:

(1) Calculation of the histogram (Figure 4) and determination of the index number (index) of the maximum pixel intensity $\left(\mathrm{im}_{\mathrm{Max}}\right)$.

(2) Calculation of the maximum histogram value $(\mathrm{Max} H)$.

(3) Decreasing the index until reaching $\mathrm{MaxH} / 3$ and setting the corresponding intensity level as the initial estimation $\left(T_{\text {calcest }}\right)$ for calculation of calcium threshold $\left(T_{\text {calc }}\right)$.

(4) Determination of the dynamic range of the image.

(i) Starting from the first bin of the histogram, the amount of pixels in each bin was counted until reaching half of the total number of pixels.

(ii) The index number of the histogram bin where the algorithm stopped corresponds to the dynamic range (DR) of the image.

(5) Calculation of the calcium threshold $\left(T_{\text {calc }}\right)$ for DR $>$ $0.7 * \mathrm{im}_{\text {Max }}$ (brighter images) by (1) and $T_{\text {calc }}$ for DR $<$ $0.7 * \mathrm{im}_{\text {Max }}$ was calculated by (2):

$$
\begin{aligned}
& T_{\text {calc }}=T_{\text {calcest }}+\left(\mathrm{im}_{\text {Max }}-T_{\text {calcest }}\right) * 0.5, \\
& T_{\text {calc }}=T_{\text {calcest }}+\left(\mathrm{im}_{\text {Max }}-T_{\text {calcest }}\right) * 0.2 .
\end{aligned}
$$

An example is given in Figure 5. The calcified pixels on grayscale image (Figure 5(a)) are detected and given a blue color (Figure 5(b)).

2.5. Computation Time. Computation time was defined as the time between the visualization of the final cropped image and the display of the measurement of the AVA size. For manual measurements it included the time required for the user to trace the orifice perimeter and the calculation of the selected area. For the semiautomatic measurements it included the selection of the seed points by the user and the computation of AVA based on the semiautomatic segmentation results. The time was measured internally by the developed software tool and displayed when the AVA size measurement was finished.

2.6. Validation and Statistical Analysis. Relative differences between the measurements were calculated to determine (1) the intraobserver variability of the semiautomatic measurements and (2) the intraobserver variability of the manual measurements. Relative difference was calculated as follows:

$$
\text { Relative difference }=\frac{\text { Absolute difference } * 100}{\text { Mean of the measurements }} .
$$

Differences between TTE and MDCT manual and semiautomatic measurements were assessed by Bland-Altman plots. Statistical analyses were performed using IBM SPSS Statistics version 20.0.0.1 (SPSS Inc., Chicago, USA). 


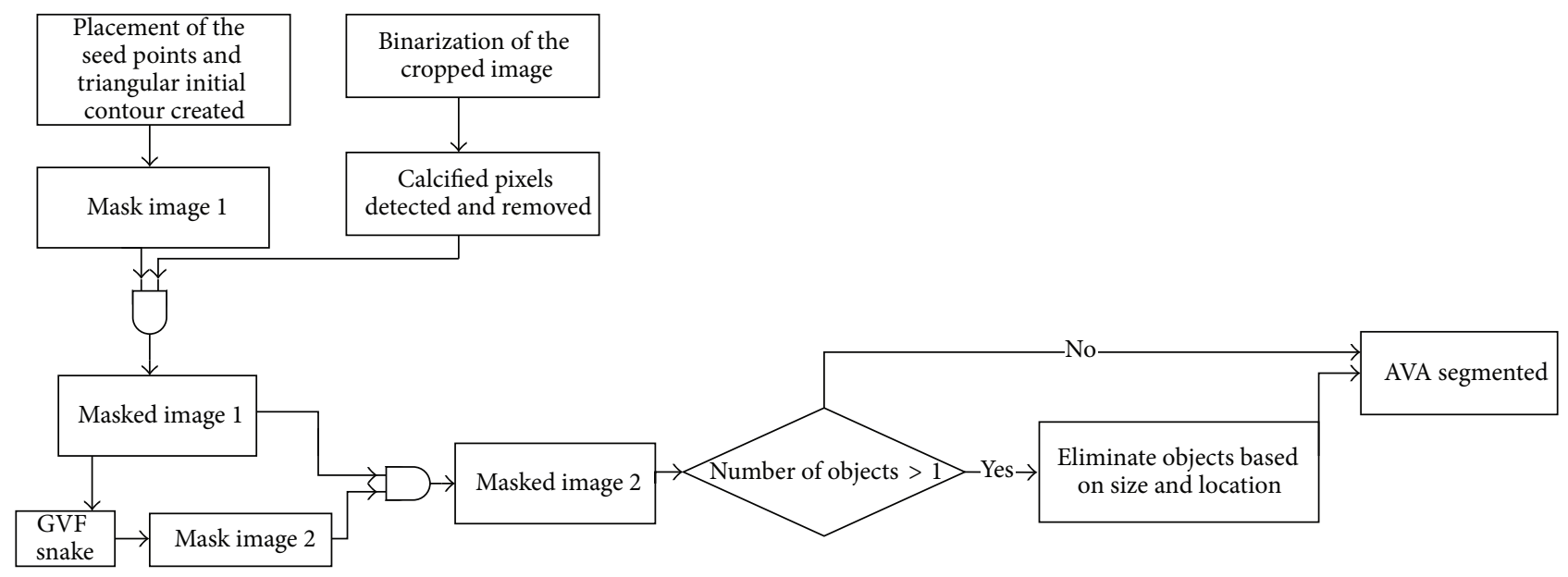

FIgURE 3: The flowchart of the AVA segmentation algorithm.

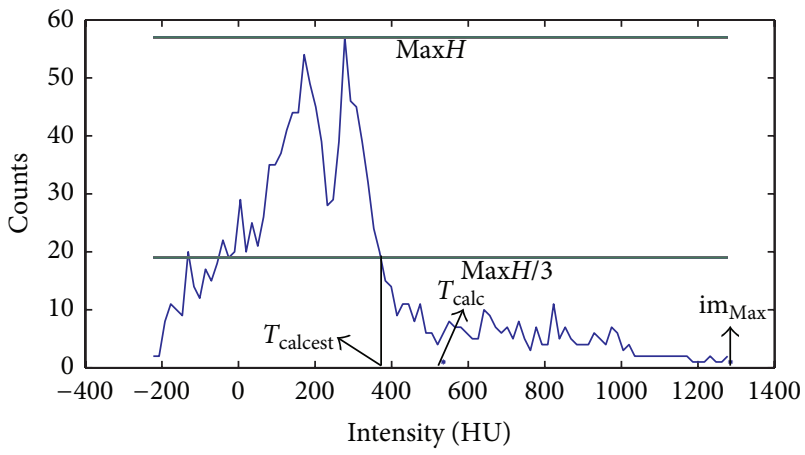

FIgURE 4: The histogram of the grayscale image.

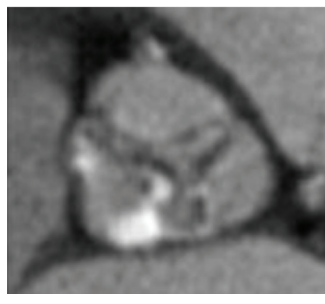

(a)

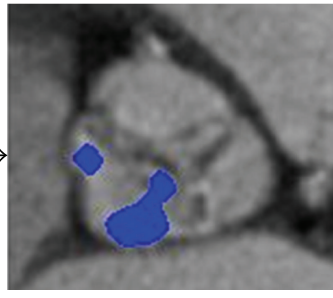

(b)
FIGURE 5: Example of the calcium detection algorithm.

\section{Results}

3.1. Segmentation Results. Aortic valve areas as measured manually and semiautomatically are listed in Table 1 . Sample results of the semiautomatic segmentation are given in Figure 6. Semiautomatic segmentation of AVA was achieved successfully for both calcified (Figures 6(a), 6(b), and 6(d)) and noncalcified aortic valves (Figure 6(c)). The output result of a segmentation and computation time for a sample image is given in Figure 6(e) showing one part of the graphic user interface.

3.2. Computation Time. The computation times of both observers were shorter for the semiautomatic measurements.
TABLE 1: Manual and semiautomatic AVA measurements.

\begin{tabular}{ll}
\hline & Mean \pm SD \\
\hline Observer 1, manual measurements $\left(\mathrm{cm}^{2}\right)$ & $0.88 \pm 0.23$ \\
Observer 1, semiautomatic measurements $\left(\mathrm{cm}^{2}\right)$ & $0.85 \pm 0.15$ \\
Observer 2, manual measurements $\left(\mathrm{cm}^{2}\right)$ & $0.98 \pm 0.29$ \\
Observer 2, semiautomatic measurements $\left(\mathrm{cm}^{2}\right)$ & $0.82 \pm 0.18$ \\
\hline
\end{tabular}

TABLE 2: Observer variability.

\begin{tabular}{lc}
\hline & Relative difference (\%) \\
\hline Intraobserver variability, manual & $8.4 \pm 7.1$ \\
Intraobserver variability, semiautomatic & $5.8 \pm 4.5$ \\
Interobserver variability, manual & $27.6 \pm 16.0$ \\
Interobserver variability, semiautomatic & $16.8 \pm 12.7$ \\
\hline
\end{tabular}

Manual measurements took $18.85 \pm 5.66$ seconds and $16.69 \pm$ 3.69 seconds for observer 1 and observer 2, respectively. Semiautomatic measurements were $5.06 \pm 0.72$ (observer 1) and $6.68 \pm 1.79$ seconds (observer 2 ).

3.3. Observer Variability. Differences in intraobserver variability of manual and semiautomatic measurements are listed in Table 2. Both intra- and interobserver variability were lower for semiautomatic measurements.

\subsection{Comparing Manual and Semiautomatic Measurements} with TTE. Comparisons of the manual and semiautomatic measurements with TTE results were performed using BlandAltman plots; mean difference between TTE and MDCT results was -0.19 (95\% CI: -0.74 to 0.34$) \mathrm{cm}^{2}$ for manual and -0.10 (95\% CI: -0.45 to 0.25$) \mathrm{cm}^{2}$ for semiautomatic measurements (Figures 7 and 8 ). The differences were significantly different from $0(p=0.001$ for manual and $p=$ 0.007 for semiautomatic measurements) indicating a bias. Both mean difference and the confidence interval are smaller in the comparison of TTE and semiautomatic measurements which indicates that semiautomatic measurements are closer to the TTE measurements than to the manual measurements. 


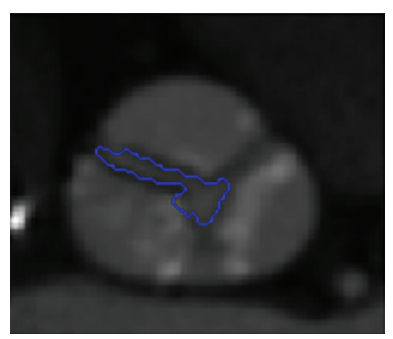

(a)

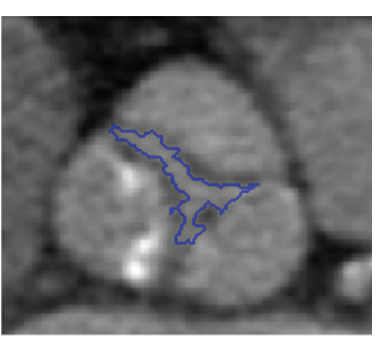

(b)

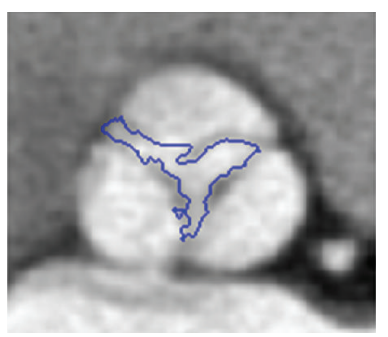

(c)

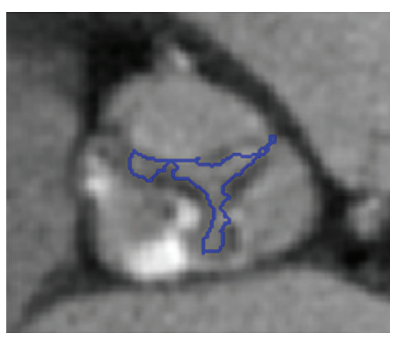

(d)

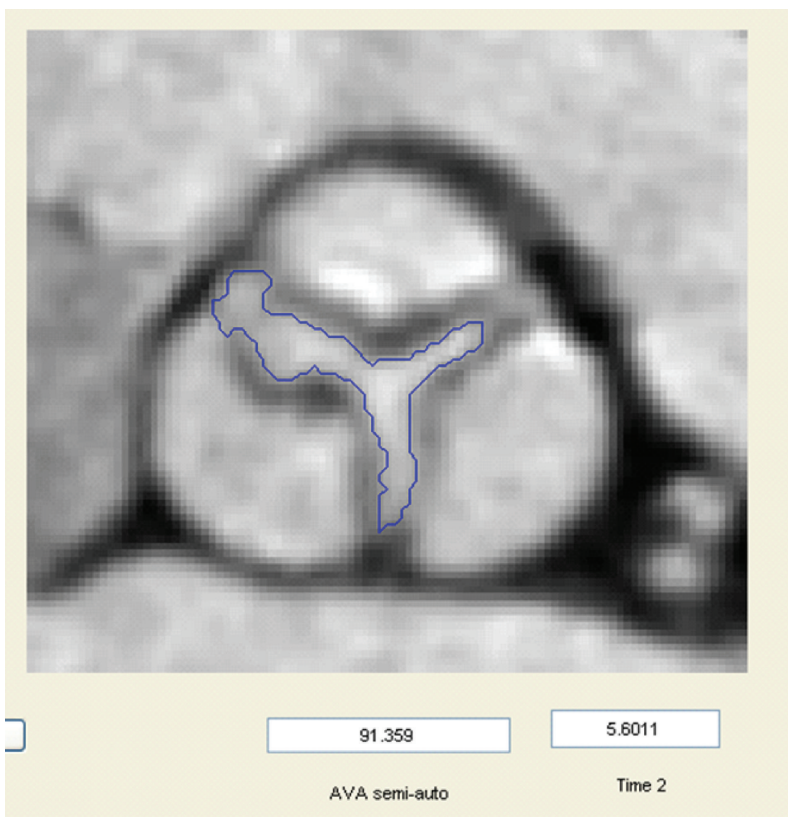

(e)

FiguRE 6: Results of semiautomatic measurements on various images (a-d). Result on graphic user interface (e).

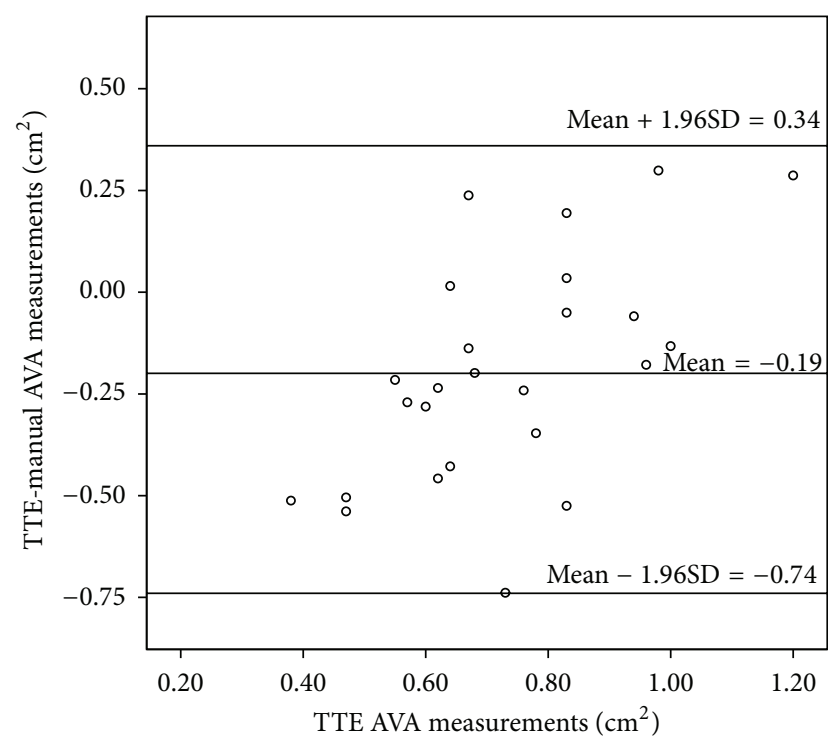

FIgURE 7: Bland-Altman plot between the TTE and manual AVA measurements.

\section{Discussion}

4.1. Research Summary. In this study we propose a semiautomatic segmentation technique to measure the AVA and compared it with the manual segmentation using TTE measurements as the reference standard. The focus of the study was to investigate whether the repeatability and reproducibility of the AVA measurements can be improved with the semiautomatic segmentation along with an improvement in computation time. First of all, our results show that semiautomatic measurements are closer to the reference TTE measurements. Furthermore the intra- and interobserver variations are lower for the semiautomatic measurements compared to manual measurements. Finally semiautomatic measurements are more than 10 seconds faster than the manual measurements.

4.2. Previous Studies and Current Study. TTE is currently the most widely used imaging modality to measure the AVA. The continuity equation, which is used to calculate the AVA based on 2D TTE data, assumes that the LVOT has a circular shape. 


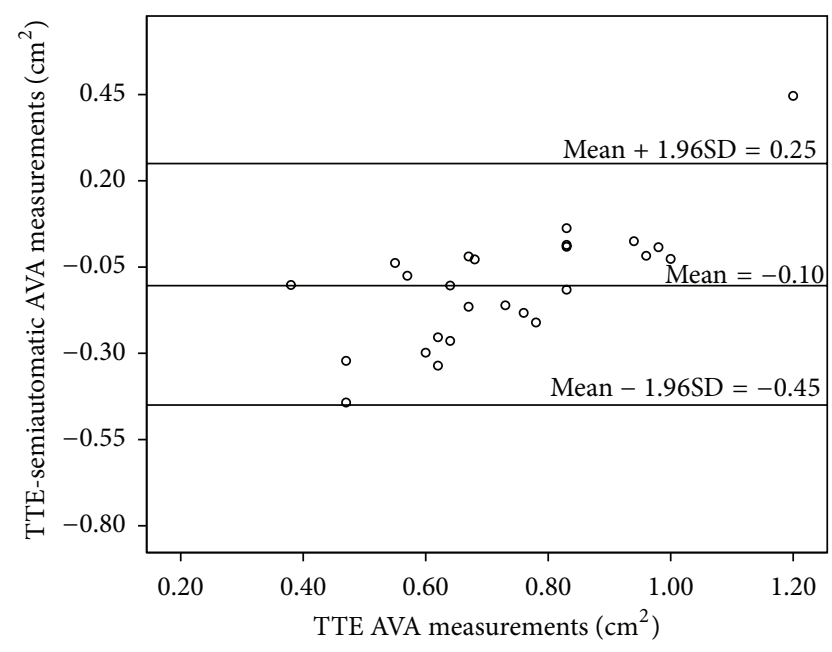

Figure 8: Bland-Altman plot between the TTE and semiautomatic AVA measurements.

A recent study showed that this assumption might cause underestimation of the AVA [30]. TTE was compared to CT in several studies. Larsen et al. observed 6\% and 16\% intraand interobserver variability for MDCT measurements on patient with broad severity of AS. Meanwhile the intra- and interobserver variability were $13 \%$ and $19 \%$ for the TTE measurements [31]. In our study the interobserver variability of semiautomatic measurements was $16 \%$ in the measurements on the patients with severe AS. Lembcke et al. conducted a study with 160 patients using 64-MDCT and TTE. They found $0.17 \pm 0.24 \mathrm{~cm}^{2}$ mean difference between MDCT and TTE measurements [32]. In our study we observed $0.19 \pm 0.27 \mathrm{~cm}^{2}$ and $0.10 \pm 0.18 \mathrm{~cm}^{2}$ mean differences in the comparisons of TTE with manual and semiautomatic MDCT measurements, respectively.

Even though (semi)automatic quantification of the aortic root dimensions such as aortic annulus, Sinus of Valsalva, and sinotubular junction using CT data has already been available in the literature [33] there is a paucity of data about (semi)automatic quantification of the AVA using CT images. Previous research already showed that echocardiography underestimates that the AVA and CT planimetric measurements are closer to the real AVA. Moreover CT is the modality used for measuring the aortic valve calcium score which associated with AS. All of these reasons make CT the method of choice. However, planimetric CT measurement of the AVA is currently performed manually, which is user dependent and time consuming. Our results demonstrate the feasibility of developing an algorithm for semiautomatic quantitative measurements of AVA in order to reduce observer variability and the time spent on the measurements. Moreover this technique is shown to also work on the target population of AS patients with a significant calcium load. The calcified regions should be detected and the opening area should be segmented excluding the calcified area. A calcification threshold is needed in order to detect the pixels belonging to the calcified region. However, in virtually all CT scans made for preoperative evaluation in patients with aortic stenosis a contrast agent is injected which makes it impossible to set a fixed calcification threshold. To overcome this issue, an algorithm was developed to calculate the calcification threshold for each CTA image individually.

The ultimate goal of fully automatic user independent segmentation was not achieved and user selection of three seed points is still required in the semiautomatic segmentation. Main reason for this is that the image quality with the current CT technology does not allow making the AVA segmentation fully automatic due to unclear object (AV) boundaries in some cases. The GVF snake was the method of choice since the snake algorithm works in cases where some parts of the object boundaries are not clear. A possible solution to make the segmentation less user dependent could be to develop an algorithm which can detect parts of the AVA boundaries (semi)automatically and interpolate the rest of the object boundary. Further developments in CT technology with higher spatial resolution and less calcium artifacts might also help to achieve the goal of fully automatic segmentation of the AVA. Our results show that CT based AVA segmentation can be achieved with less user dependence and as a result a higher reproducibility and less time consuming measurements of AVA segmentation were obtained.

4.3. Limitations. A possible limitation of our study was that the users were not asked to rechoose the phase and opening plane on which to measure the AVA. However, this choice was made to eliminate the user interference in the measurement results in order to really test the accuracy of the developed algorithm. Another limitation of this study is the selected patient group. We studied a relatively small sample of patients with varying delay between TTE and CT imaging and all subjects had severe AS (mean AVA smaller than $1.0 \mathrm{~cm}^{2}$ ). Future work will have to be carried out in larger cohorts containing subject with varying degrees of AS. Also, we cannot rule out that differences in AVA can be attributed to differences in area over time as opposed to difference inherent to the imaging techniques used. The time difference between the CT and ultrasound measurements was more than 100 days for 6 patients. A final limitation is having 2 different measurement techniques using the TTE and MDCT data. In MDCT measurements we had the direct measurements using the planimetric image of the AVA; on the other hand AVA was measured indirectly by the flow information gathered by the TTE. This difference between the measurement techniques led to the variation between the TTE and MDCT manual and semiautomatic measurements. Therefore further work required to determine what the clinical followup should be based on MDCT measurements.

4.4. Implications. Studies comparing the use of CT and echocardiography found that CT can be an alternative to the current gold standard echocardiography in the quantification of AVA [26, 27]. Our study has some implications in semiautomatic quantification of AVA on the CT images. First of all the intra- and interobserver variability of semiautomatic measurements are better than the manual measurements. These results imply that the variation caused by the user interaction is decreased by using the semiautomatic software, 
which is desirable for quantitative assessment of medical images. Moreover semiautomatic software provides a faster calculation of the AVA in comparison with the manual measurements. Faster measurements decrease the workload. The comparison of manual and semiautomatic CT measurements with the current standard TTE measurements revealed that semiautomatic measurements are closer to the TTE measurements. If the standard modality for measuring the AVA will switch from echocardiography to CT, semiautomatic measurements can serve as a better option in comparison to the manual measurements due to the smaller difference between the TTE and semiautomatic measurements.

\section{Conclusion}

In this study a semiautomatic segmentation technique that can be used in AVA segmentation is proposed. Based on preliminary results the algorithm provides adequate segmentation of representative images, also those including severe calcification, and provides a faster, more accurate, and more reproducible AVA segmentation compared to the currently used manual segmentation.

\section{Disclosure}

The authors would like to state that there is no relationship with industry in this study. Recently, R. P. J. Budde moved to the Erasmus Medical Center, Rotterdam, The Netherlands.

\section{Conflict of Interests}

The authors declare that there is no conflict of interests regarding the publication of this paper.

\section{References}

[1] M. Lindroos, M. Kupari, J. Heikkila, and R. Tilvis, "Prevalence of aortic valve abnormalities in the elderly: an echocardiographic study of a random population sample," Journal of the American College of Cardiology, vol. 21, no. 5, pp. 1220-1225, 1993.

[2] W. Grossman, D. Jones, and L. P. McLaurin, "Wall stress and patterns of hypertrophy in the human left ventricle," The Journal of Clinical Investigation, vol. 56, no. 1, pp. 56-64, 1975.

[3] American College of Cardiology/American Heart Association Task Force on Practice Guidelines, Society of Cardiovascular Anesthesiologists, Society for Cardiovascular Angiography and Interventions et al., "ACC/AHA 2006 guidelines for the management of patients with valvular heart disease: a report of the American College of Cardiology/American Heart Association Task Force on Practice Guidelines (writing committee to revise the 1998 Guidelines for the Management of Patients With Valvular Heart Disease): developed in collaboration with the Society of Cardiovascular Anesthesiologists: endorsed by the Society for Cardiovascular Angiography and Interventions and the Society of Thoracic Surgeons," Circulation, vol. 114, no. 5, pp. e84-e231, 2006.

[4] B. Iung, A. Cachier, G. Baron et al., "Decision-making in elderly patients with severe aortic stenosis: why are so many denied surgery?" European Heart Journal, vol. 26, no. 24, pp. 2714-2720, 2005.
[5] H. R. Andersen, L. L. Knudsen, and J. M. Hasenkam, "Transluminal implantation of artificial heart valves. Description of a new expandable aortic valve and initial results with implantation by catheter technique in closed chest pigs," European Heart Journal, vol. 13, no. 5, pp. 704-708, 1992.

[6] A. Cribier, H. Eltchaninoff, C. Tron et al., "Early experience with percutaneous transcatheter implantation of heart valve prosthesis for the treatment of end-stage inoperable patients with calcific aortic stenosis," Journal of the American College of Cardiology, vol. 43, no. 4, pp. 698-703, 2004.

[7] E. Grube, J. C. Laborde, U. Gerckens et al., "Percutaneous implantation of the CoreValve self-expanding valve prosthesis in high-risk patients with aortic valve disease: the siegburg firstin-man study," Circulation, vol. 114, no. 15, pp. 1616-1624, 2006.

[8] E. Grube, G. Schuler, L. Buellesfeld et al., "Percutaneous aortic valve replacement for severe aortic stenosis in highrisk patients using the second- and current third-generation self-expanding CoreValve prosthesis: device success and 30-day clinical outcome," Journal of the American College of Cardiology, vol. 50, no. 1, pp. 69-76, 2007.

[9] S. V. Lichtenstein, A. Cheung, J. Ye et al., "Transapical transcatheter aortic valve implantation in humans: initial clinical experience," Circulation, vol. 114, no. 6, pp. 591-596, 2006.

[10] J. G. Webb, M. Chandavimol, C. R. Thompson et al., "Percutaneous aortic valve implantation retrograde from the femoral artery," Circulation, vol. 113, no. 6, pp. 842-850, 2006.

[11] J. G. Webb, S. Pasupati, K. Humphries et al., "Percutaneous transarterial aortic valve replacement in selected high-risk patients with aortic stenosis," Circulation, vol. 116, no. 7, pp. 755763, 2007.

[12] N. Saikrishnan, G. Kumar, F. J. Sawaya, S. Lerakis, and A. P. Yoganathan, "Accurate assessment of aortic stenosis: a review of diagnostic modalities and hemodynamics," Circulation, vol. 129, no. 2, pp. 244-253, 2014.

[13] J. K. Willmann, D. Weishaupt, M. Lachat et al., "Electrocardiographically gated multi-detector row CT for assessment of valvular morphology and calcification in aortic stenosis," Radiology, vol. 225, no. 1, pp. 120-128, 2002.

[14] G. M. Feuchtner, S. Müller, J. Bonatti et al., "Sixty-four slice CT evaluation of aortic stenosis using planimetry of the aortic valve area," American Journal of Roentgenology, vol. 189, no. 1, pp. 197203, 2007.

[15] E. Bouvier, D. Logeart, J.-L. Sablayrolles et al., "Diagnosis of aortic valvular stenosis by multislice cardiac computed tomography," European Heart Journal, vol. 27, no. 24, pp. 30333038, 2006.

[16] R. C. Gilkeson, A. H. Markowitz, A. Balgude, and P. B. Sachs, "MDCT evaluation of aortic valvular disease," American Journal of Roentgenology, vol. 186, no. 2, pp. 350-360, 2006.

[17] S. J. Cowell, D. E. Newby, J. Burton et al., "Aortic valve calcification on computed tomography predicts the severity of aortic stenosis," Clinical Radiology, vol. 58, no. 9, pp. 712-716, 2003.

[18] R. Koos, A. H. Mahnken, A. M. Sinha, J. E. Wildberger, R. Hoffmann, and H. P. Kühl, "Aortic valve calcification as a marker for aortic stenosis severity: assessment on 16-MDCT," American Journal of Roentgenology, vol. 183, no. 6, pp. 1813-1818, 2004.

[19] G. J. Morgan-Hughes, P. E. Owens, C. A. Roobottom, and A. J. Marshall, "Three dimensional volume quantification of aortic valve calcification using multislice computed tomography," Heart, vol. 89, no. 10, pp. 1191-1194, 2003. 
[20] O. Bruder, M. Jochims, P. Hunold et al., "Comparison of aortic valve area measured by magnetic resonance imaging and dualsource computed tomography," Acta Radiologica, vol. 50, no. 6, pp. 645-651, 2009.

[21] A. Lembcke, D. E. Kivelitz, A. C. Borges et al., "Quantification of aortic valve stenosis: head-to-head comparison of 64-slice spiral computed tomography with transesophageal and transthoracic echocardiography and cardiac catheterization," Investigative Radiology, vol. 44, no. 1, pp. 7-14, 2009.

[22] E. J. Weinberg and M. R. Kaazempur Mofrad, "A multiscale computational comparison of the bicuspid and tricuspid aortic valves in relation to calcific aortic stenosis," Journal of Biomechanics, vol. 41, no. 16, pp. 3482-3487, 2008.

[23] F. Chenot, P. Montant, C. Goffinet et al., "Evaluation of anatomic valve opening and leaflet morphology in aortic valve bioprosthesis by using multidetector CT: comparison with transthoracic echocardiography," Radiology, vol. 255, no. 2, pp. 377-385, 2010.

[24] F. A. Flachskampf, "Severe aortic stenosis with low gradient and apparently preserved left ventricular systolic function-underrecognized or overdiagnosed?" European Heart Journal, vol. 29, no. 8, pp. 966-968, 2008.

[25] K. K. Poh, R. A. Levine, J. Solis et al., "Assessing aortic valve area in aortic stenosis by continuity equation: a novel approach using real-time three-dimensional echocardiography," European Heart Journal, vol. 29, no. 20, pp. 2526-2535, 2008.

[26] O. Klass, M. J. Walker, M. E. Olszewski et al., "Quantification of aortic valve area at 256-slice computed tomography: comparison with transesophageal echocardiography and cardiac catheterization in subjects with high-grade aortic valve stenosis prior to percutaneous valve replacement," European Journal of Radiology, vol. 80, no. 1, pp. 151-157, 2011.

[27] V. Delgado and J. J. Bax, "Classical methods to measure aortic valve area in the era of new invasive therapies: still accurate enough?” International Journal of Cardiovascular Imaging, vol. 25, no. 2, pp. 183-185, 2009.

[28] T. Gaspar, S. Adawi, R. Sachner et al., "Three-dimensional imaging of the left ventricular outflow tract: impact on aortic valve area estimation by the continuity equation," Journal of the American Society of Echocardiography, vol. 25, no. 7, pp. 749-757, 2012.

[29] C. Xu and J. L. Prince, "Snakes, shapes, and gradient vector flow," IEEE Transactions on Image Processing, vol. 7, no. 3, pp. 359-369, 1998.

[30] H. Utsunomiya, H. Yamamoto, J. Horiguchi et al., "Underestimation of aortic valve area in calcified aortic valve disease: effects of left ventricular outflow tract ellipticity," International Journal of Cardiology, vol. 157, no. 3, pp. 347-353, 2012.

[31] L. H. Larsen, K. F. Kofoed, H. G. Carstensen et al., "Aortic valve area assessed with 320-detector computed tomography: comparison with transthoracic echocardiography," International Journal of Cardiovascular Imaging, vol. 30, no. 1, pp. 165-173, 2014.

[32] A. Lembcke, M. Woinke, A. C. Borges et al., "Grading of aortic valve stenosis at 64-Slice spiral computed tomography: comparison with transthoracic echocardiography and calibration against cardiac catheterization," Investigative Radiology, vol. 44, no. 6, pp. 360-368, 2009.

[33] V. Delgado, A. C. T. Ng, J. D. Schuijf et al., "Automated assessment of the aortic root dimensions with multidetector row computed tomography," Annals of Thoracic Surgery, vol. 91, no. 3, pp. 716-723, 2011. 


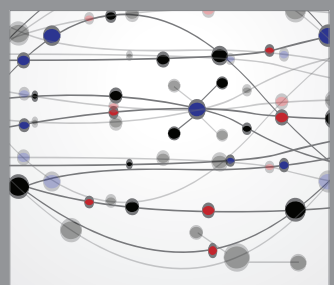

The Scientific World Journal
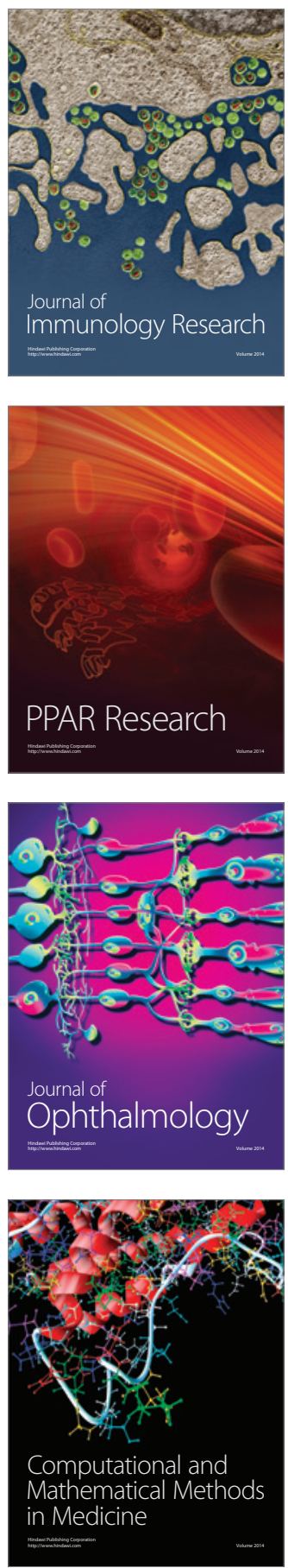

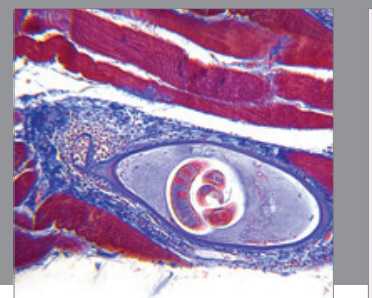

Gastroenterology

Research and Practice
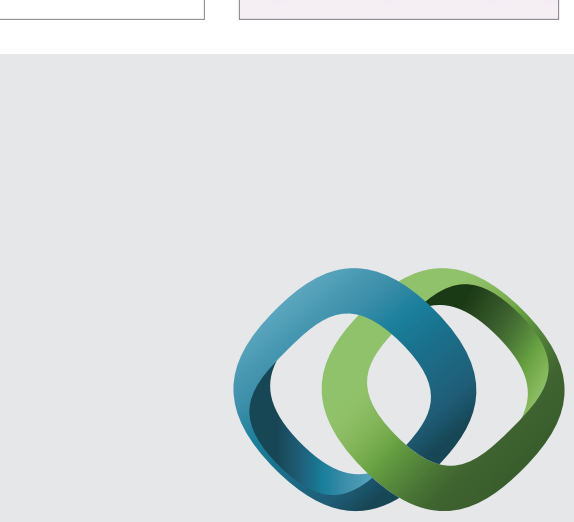

\section{Hindawi}

Submit your manuscripts at

http://www.hindawi.com
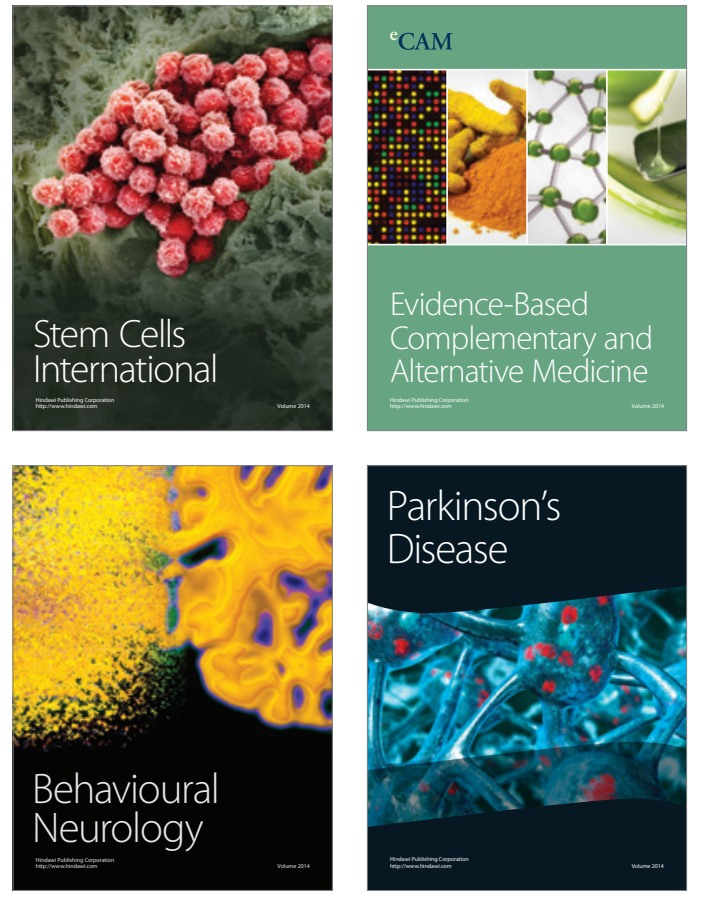
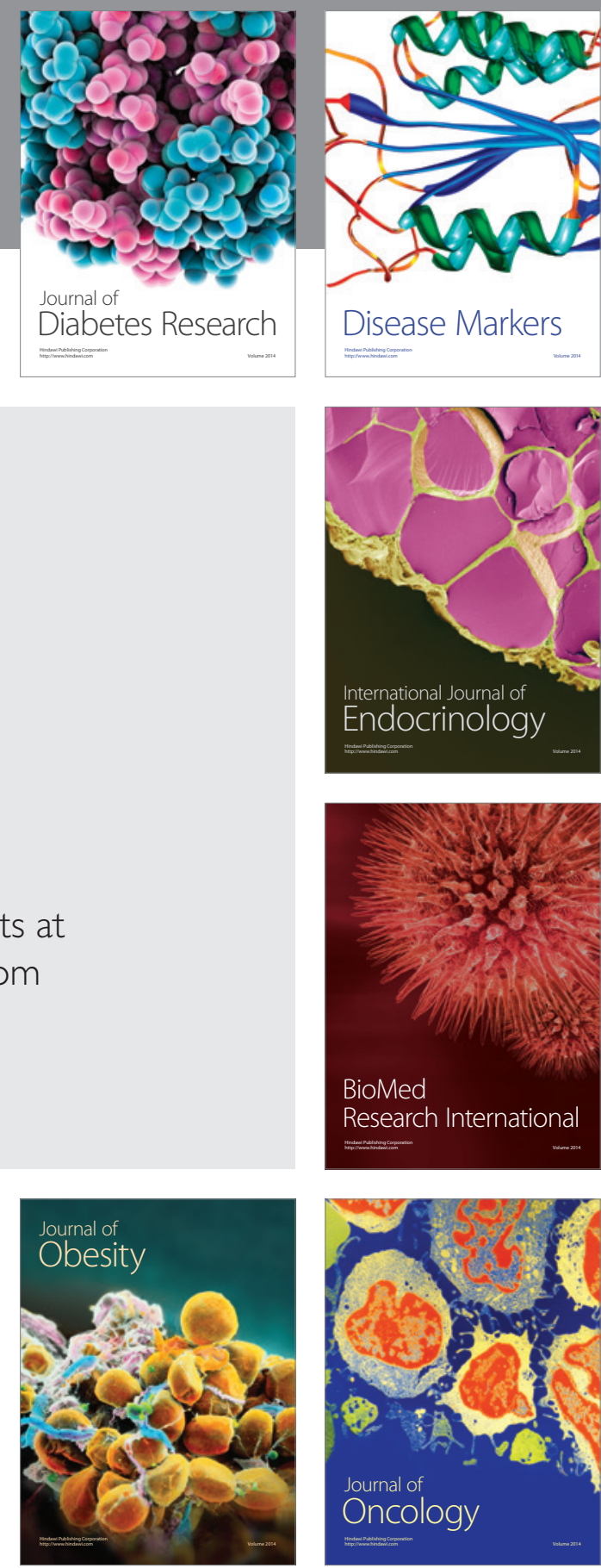

Disease Markers
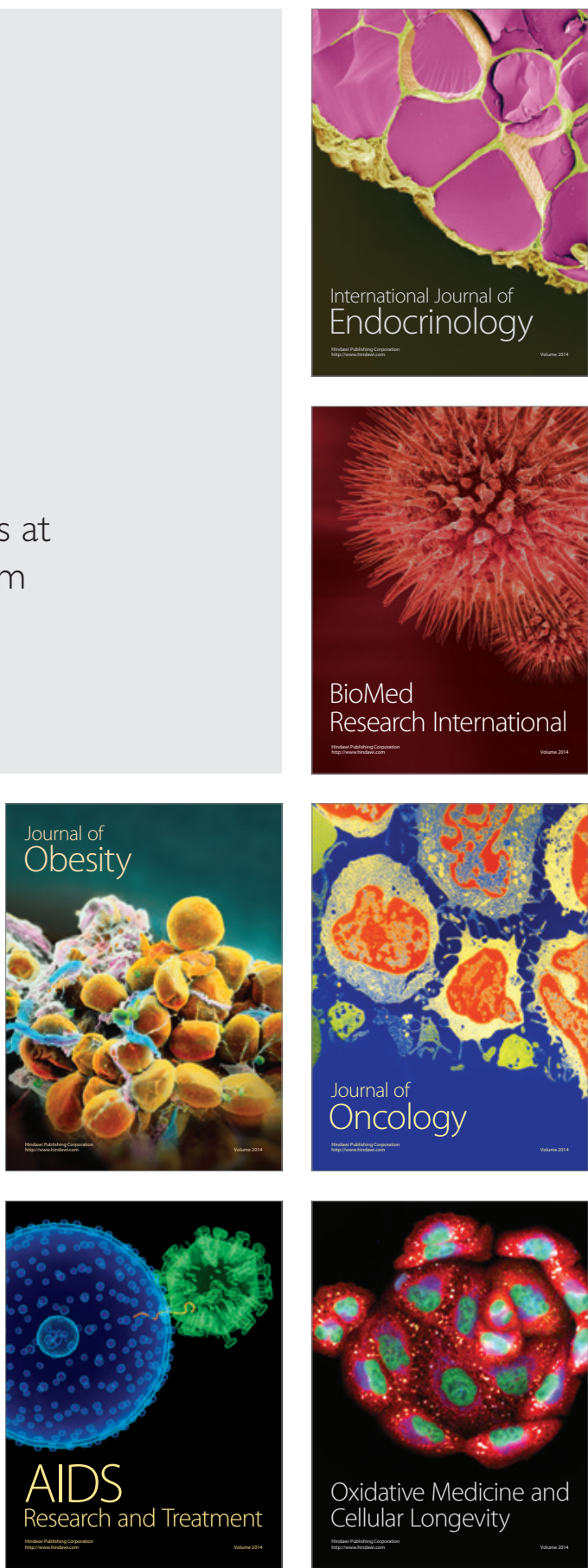